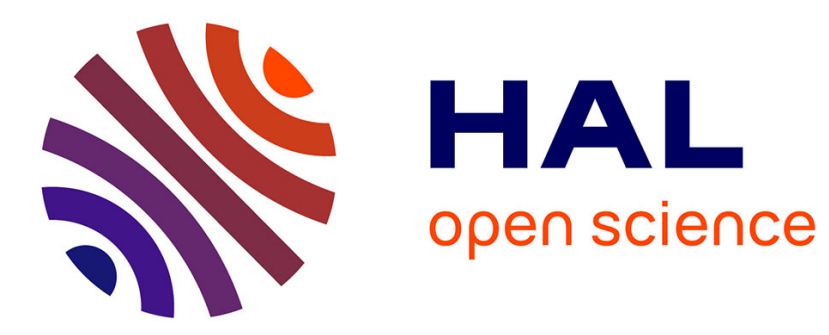

\title{
Steering Focusing Waves in a Reverberation Chamber with Generalized Time Reversal
}

\author{
Andrea Cozza, Florian Monsef
}

\section{To cite this version:}

Andrea Cozza, Florian Monsef. Steering Focusing Waves in a Reverberation Chamber with Generalized Time Reversal. IEEE Transactions on Antennas and Propagation, 2016, 65 (3), pp.1349 - 1356. 10.1109/TAP.2016.2645788 . hal-01377945

\section{HAL Id: hal-01377945}

https://hal-centralesupelec.archives-ouvertes.fr/hal-01377945

\author{
Submitted on 7 Oct 2016
}

HAL is a multi-disciplinary open access archive for the deposit and dissemination of scientific research documents, whether they are published or not. The documents may come from teaching and research institutions in France or abroad, or from public or private research centers.
L'archive ouverte pluridisciplinaire HAL, est destinée au dépôt et à la diffusion de documents scientifiques de niveau recherche, publiés ou non, émanant des établissements d'enseignement et de recherche français ou étrangers, des laboratoires publics ou privés. 


\title{
Steering Focusing Waves in a Reverberation Chamber with Generalized Time Reversal
}

\author{
Andrea Cozza, Senior Member, IEEE, Florian Monsef, Member, IEEE
}

\begin{abstract}
Generalized time reversal was introduced in a previous paper from a theoretical point of view. In this paper experiments are conducted to demonstrate its application to a reverberation chamber, as a method for generating coherent wavefronts in a medium displaying random propagation. Wavefronts thus generated were sampled over a planar surface, confirming that they propagate as if in a free-space environment. The accuracy with which they match their free-space counterpart is not affected by changing their features, e.g., direction of arrival and focus. These results prove that a single excitation antenna can generate complex wavefronts when coupled to diffusive wave propagation.
\end{abstract}

Index Terms-Time reversal, diffusive media, reverberation chamber, wavefront synthesis.

\section{BACKGROUND AND MOTIVATIONS}

Time reversal (TR) was first introduced in acoustics as a method for generating wavefronts focusing back the field distribution initially radiated by a source [1]. Its intrinsical ability to compensate distortions introduced by heterogeneous and complex media with no need for active equalization is perhaps its most striking feature [2]-[4]. Indeed, beam-forming methods require a priori information about the propagation of waves in a given medium; this need is avoided in TR applications by the fact that the wave radiated by the source (which would afterwards be focused back at it) acts as a sort of medium sounding, and thus brings in itself information about the medium. Moreover, contrarily to methods based on inverse problems, TR is not affected by any ill-posedness, as opposed to dereverberation methods [5]. Other beam-forming techniques, such as wave field synthesis [6], require a very large number of synchronous sources, which would typically be prohibitively expensive at microwave frequencies.

Still, TR suffers from an obvious drawback: focusing waves cannot be synthesized arbitrarily, as they can only reproduce previously radiated wavefronts. This is particularly problematic when thinking about applications of TR for testing the response to impinging wavefronts of a device under test.

Generalized TR (GTR) was first tested in an embryonic version in [7], [8], where direct wavefront synthesis was not yet formalized, as later done from a theoretical point of view in [9]. It is based on the use of the equivalence theorem as a way of avoiding the introduction of a source, in order to directly synthesize signals capable of generating focusing

Part of the work here reported was funded by the French National Research Agency through the grant ANR-12-ASTR-0005, MIMOCHIC project, within the framework of the 2012 ASTRID program. The design and manufacturing of the robot were funded by Digiteo, under the AUTOTREC project.

A. Cozza and F. Monsef are with PIEM | GeePs, UMR 8507, CentraleSupelec, Univ Paris Sud, UPMC, CNRS, 1 rue Joliot-Curie, 91192 Gif-sur-Yvette, France. Contact e-mail: andrea.cozza@ieee. org. wavefronts with arbitrary features. GTR is entirely dependent on the availability of a medium capable of supporting diffusefield propagation, a statistically isotropic case of multipath propagation, also known as Rayleigh diffusion. Reverberation chambers comply with these requirements and bring at the same time a further advantage, energy efficiency, being closed systems with weak losses; when used with TR signals their efficiency can be further increased [10].

This paper shows validation results obtained with a fully automated system. Experimental results of wavefronts generated within a reverberation chamber are compared to theoretically computed distributions. Sequences of wavefronts varying in the position of their focal region and direction of arrival are generated, confirming that the diffusive nature of the medium ensures an excellent reproducibility of wavefronts at any position.

The conclusions of this study imply that GTR can accurately generate arbitrary pulsed focusing wavefronts with a single antenna. Moreover, generating similar focusing wavefronts in open media could require a potentially large number of sources, depending on the angular spectrum of the wavefront, i.e., its focusing power. These sources would need to be excited coherently over a relatively large bandwidth, an operation which is complex and expensive, as it requires synchronizing a number of modulators or signal generators, which can hardly handle high peak-power levels, were them required.

Applications of GTR-based synthesis are currently in progress and range from hardening shielded systems to high-power microwaves, imaging and generating stable highintensity fields without the statistical uncertainty found in standard reverberation chambers [10].

The paper is organized as follows. GTR is briefly explained in Sec. II, as an intuitive application of first principles wellknown in electromagnetics. Sec. III provides some details about the way wavefront-related data is generated. The experimental setup is described in Sec. IV, while Sec. V explains how generated fields are imaged. Reproducibility and robustness to varying conditions are tested and commented in Sec. VI.

\section{A SHORT SUMMARY OF GTR}

This section presents a very short description of how GTR works. The simplest way to understand it is by going through the different steps at its origin. The interested reader can refer to [9] for a much more detailed discussion.

Suppose a source radiates a free-space field distribution $\boldsymbol{E}_{s}(\boldsymbol{r}, t)$. When excited within a medium supporting diffusive propagation, the resulting field distribution would be 


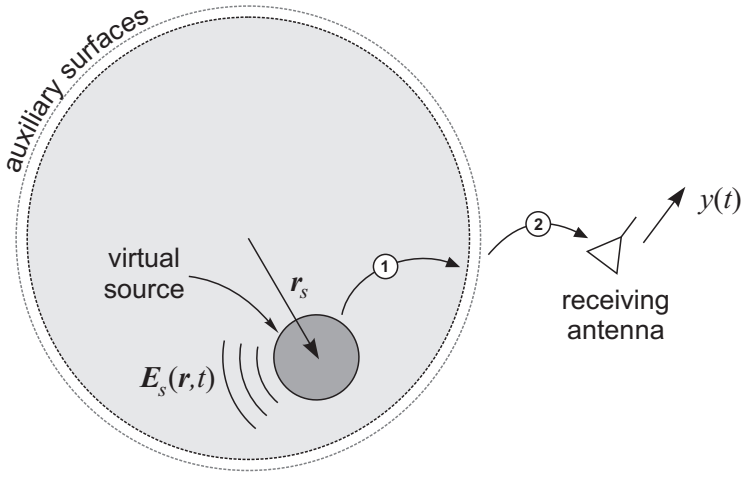

Fig. 1: Relevant quantities in the derivation of direct synthesis of excitation signals with GTR. The auxiliary surfaces are shown to act as an interface between the virtual source radiation (1) and its propagation through the medium (2).

underpinned by a rich plane-wave spectrum, due to a large number of scattering events over its boundaries [11]-[13]. Standard time-reversal would require measuring the signal $y(t)$ received by a further antenna present in the medium [1], [14]. Injecting the time-reversed signal $x(t)=y(-t)$ into what was the receiving antenna, eventually corrected for its dispersive response, can generate a close replica of $\boldsymbol{E}_{s}(\boldsymbol{r},-t)$, i.e., the converging counterpart to the originally diverging wavefront $\boldsymbol{E}_{s}(\boldsymbol{r}, t)$, stripped of its reactive, i.e., non-propagating, components. A single source can support an entire wavefront thanks to self-averaging ensured by the multipath propagation of the medium, if excitation bandwidths cover several coherence bandwidths of the medium [15]. Stated in other terms, complex media can present frequency responses that become weakly interdependent when observed at frequencies spanning at least one coherence bandwidth. The result is the existence of degrees of freedom that can be roughly measured by taking the ratio of the bandwidth of the excitation signal divided by the coherence bandwidth of the medium. TR signals work by controlling these degrees of freedom such that at the time of focusing they cooperate to create coherent wavefronts, out of individual random contributions from each frequency. This property, known as self-averaging, is fundamentally equivalent to the mechanisms behind Van Cittert-Zernike theorem [16], where degrees of freedom are in space rather than in the frequency domain, and result in overall coherent field distribution generated from random spatial distributions.

The problem with the TR appproach is that a focusing wavefront can be generated only if a source radiates a corresponding divergent wavefront in the first place. Clearly, if several focusing wavefronts were to be generated, not only the source would have to be modified (e.g., orientation, position) and made to radiate, but also an array of sources with different characteristics may be required (e.g., directivity, polarization).

GTR bypasses this problem by getting rid of the need for physical sources. The excitation signal $x(t)$ is directly synthesized, according to the wished characteristics of the focusing wavefront. In order to do that, $y(t)$ is obtained in two steps. With reference to Fig. 1, first the field radiated by the source is sampled over (at least) an auxiliary surface $\Sigma$. From these samples, equivalent currents are defined, capable of reproducing the outgoing field that would have reached the receiving antenna, were the source real. In the second step, $y(t)$ is computed from an assumed knowledge of the Green's functions on the medium, acting as transfer functions between the equivalent sources over $\Sigma$ and the receiving antenna, hereafter referred to as $\boldsymbol{N}_{e}(\boldsymbol{r}, \omega)$ and $\boldsymbol{N}_{m}(\boldsymbol{r}, \omega)$, respectively for the electric and magnetic field Green's functions. The result is a direct synthesis formula for the Fourier spectrum of the received signal $y(t)[9]$

$$
Y(\omega)=\int_{\Sigma} \mathrm{d} \boldsymbol{r}\left[\frac{\boldsymbol{N}_{e}(\boldsymbol{r}, \omega)}{\zeta}+\hat{\boldsymbol{r}} \times \boldsymbol{N}_{m}(\boldsymbol{r}, \omega)\right] \cdot \boldsymbol{E}_{s}(\boldsymbol{r}, \omega) .
$$

The derivation of (1) means that the auxiliary surfaces act as a relay in the synthesis of the signal $y(t)$ generated by a given virtual source. Moreover, since the field radiated by the virtual source is not sampled over $\Sigma$ for its own sake, but rather in order to propagate it to the receiver, there is no need to include reactive contributions [17], [18], due to an eventually near-field configuration. As a result, far-field representations of $\boldsymbol{E}_{s}(\boldsymbol{r}, t)$ can be used, as done in Sec. V.

If the Green's functions were known, then the first step could be accomplished numerically, with no need of a source in the first place, just by defining firsthand the field distribution expected over $\Sigma$. In fact, since the medium is reciprocal, the Green's functions can be measured by exciting the receiving antenna and sampling the electromagnetic field distribution it excites over $\Sigma$. This operation is carried out in our case by a low-perturbation robot developed in our laboratory, as discussed in Sec. IV.

In practice, weakly perturbative field probes are needed. Since most often electric-field probes have a better performance in this respect, equivalent currents can be defined from electric field samples only, if a further auxiliary surface is introduced. As demonstrated in [19], this is possible if specific weights $\left\{A_{i}\right\}, i \in[1, L]$ are applied to the field distributions sampled over each auxiliary surface $\Sigma_{l}$, yielding

$$
Y(\omega)=\zeta^{-1} \sum_{l=1}^{L} A_{l} \int_{\Sigma_{l}} \mathrm{~d} \boldsymbol{r} \boldsymbol{N}_{e}(\boldsymbol{r}, \omega) \cdot \boldsymbol{E}_{s}(\boldsymbol{r}, \omega)
$$

For the case $L=2, A_{1}=-\exp (-2 \mathrm{j} k \Delta R) /[1-$ $\exp (-2 \mathrm{j} k \Delta R)], A_{2}=-A_{1} \exp (2 \mathrm{j} k \Delta R)$, with $\Delta R$ the difference between the radii of the two spherical auxiliary surfaces, required to satisfy $\Delta R<\lambda / 2$, with $\lambda$ the working wavelength.

\section{REFERENCE FIELD DISTRIBUTIONS}

With reference to Fig. 2, the definition of the virtual source is based on a Gaussian far-field radiation pattern, with phase center $\boldsymbol{r}_{s}$

$$
\boldsymbol{E}_{s}(\boldsymbol{r}, \omega)=\hat{\varphi} G\left(\boldsymbol{r}-\boldsymbol{r}_{s}, \omega\right) \mathrm{e}^{-\psi^{2} / 2 \psi_{s}^{2}} P(\omega)
$$

and $\psi=\cos ^{-1}\left(\hat{\boldsymbol{r}}^{\prime} \cdot \hat{\boldsymbol{y}}\right)$, for an angular divergence $\psi_{s} ; G(\boldsymbol{r} . \omega)$ is the scalar Green's function for free space; the polarization is set equal to $\hat{\varphi} ; P(\omega)$ is the Fourier spectrum of $p(t)$, the time evolution of the radiated field, usually chosen to be a pulse. 


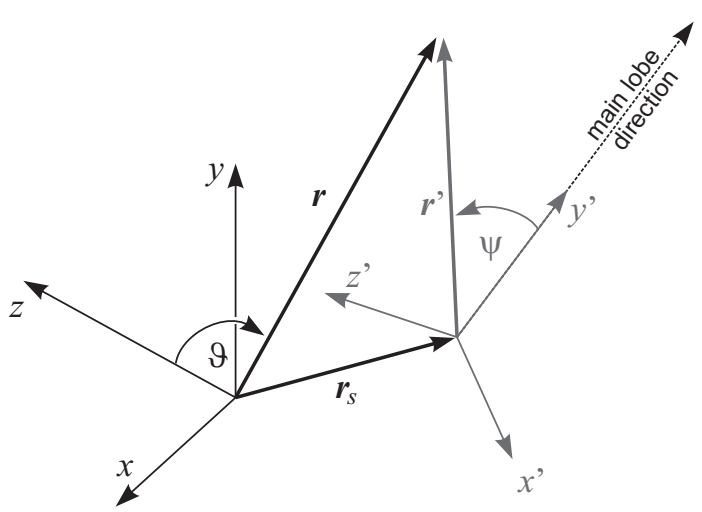

Fig. 2: Global and local reference frames for the computation of the vector field distribution at $r$, radiated by a source centered over $\boldsymbol{r}_{s}$.

A Gaussian pulse will be used throughout the paper, with a Fourier spectrum

$$
P(\omega)=\mathrm{e}^{-\left(\omega-\omega_{c}\right)^{2} / 2 B_{e}^{2}},
$$

with $B_{e}$ the equivalent bandwidth of the pulses.

In order to orient the main lobe along any direction $\hat{r}_{\text {inc }}$, with arbitrarily oriented polarization, (3) is considered as defined within the local reference frame shown in Fig. 2 in grey with primed quantities, reoriented with respect to the global reference frame through a rotation matrix $\boldsymbol{R}$.

The spherical unit vectors $\hat{\boldsymbol{u}}_{i}(\boldsymbol{r})$ of the global reference frame are juxtaposed into a matrix $\boldsymbol{U}(\boldsymbol{r})$, and the same is done for the unit vectors $\hat{\boldsymbol{u}}_{i}^{\prime}\left(\boldsymbol{r}^{\prime}\right)$ of the local reference frame into a matrix $\boldsymbol{U}^{\prime}\left(\boldsymbol{r}^{\prime}\right)$. The local-frame field $\boldsymbol{E}^{\prime}\left(\boldsymbol{r}^{\prime}\right)$ then appears to the global frame as $\boldsymbol{R} \boldsymbol{E}^{\prime}\left(\boldsymbol{r}^{\prime}\right)$ and can be decomposed into the global unit-vector representation as

$$
\boldsymbol{E}_{s}(\boldsymbol{r}, \omega)=\boldsymbol{U}^{\mathrm{T}}(\boldsymbol{r}) \boldsymbol{R} \boldsymbol{U}^{\prime}\left(\boldsymbol{r}^{\prime}\right) \boldsymbol{E}_{s}^{\prime}\left(\boldsymbol{r}^{\prime}, \omega\right),
$$

knowing that $\boldsymbol{E}_{s}^{\prime}\left(\boldsymbol{r}^{\prime}, \omega\right)=E_{s}\left(\boldsymbol{r}^{\prime}, \omega\right) \hat{\boldsymbol{\varphi}}$, as of (3); $\mathrm{T}$ is the matrix transpose.

Since (3) makes use of the scalar Green's function, it can only account for far-field radiation. This is not an issue, since the source-radiated field distribution over the auxiliary surfaces has a relay role, as recalled in Sec. II.

Eq. (3) is also needed in order to predict the reference (theoretical) field distribution that GTR is expected to produce. It can be computed by using a plane-wave spectrum (PWS) representation of the radiated field, which corresponds to the far-field radiation [18], and thus coincides with (3) as sampled over a spherical surface centered at the origin of the global reference frame. The value chosen for the radius $R$ of this test surface has no impact on the PWS computation, as long as $R$ is much larger than the radii of the auxiliary surfaces.

The refocused field distribution can then be computed by switching the direction of the plane waves from outbound to inbound, yielding

$$
\boldsymbol{E}_{\mathrm{GTR}}(\boldsymbol{r}, t)=\frac{1}{2 \pi} \int \mathrm{d} \omega \mathrm{e}^{\mathrm{j} \omega t} \int \mathrm{d} \hat{\boldsymbol{k}} \boldsymbol{E}_{s}^{*}(R \hat{\boldsymbol{k}}, \omega) \mathrm{e}^{\mathrm{j} \boldsymbol{k} \cdot \boldsymbol{r}},
$$
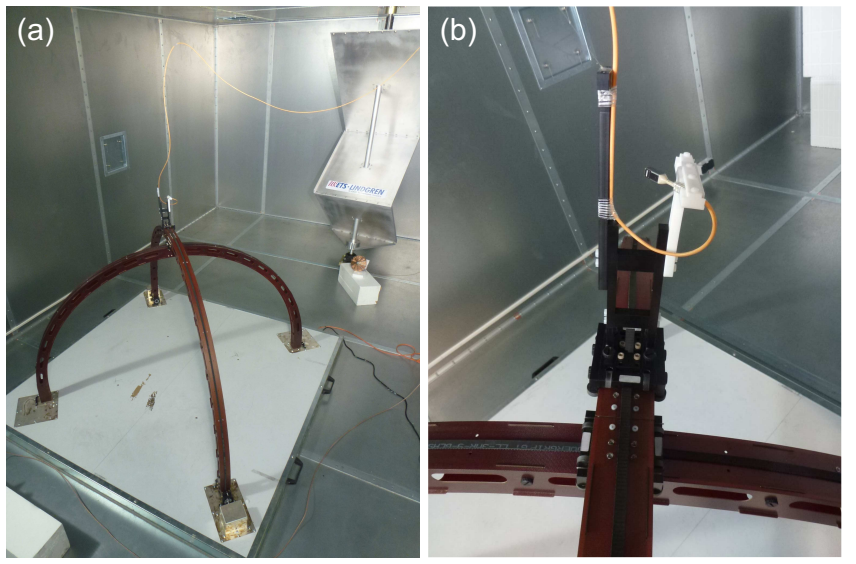

Fig. 3: The hemispherical field scanner with the excitation antenna (a) and the electro-optical probe (b) used during the experimental validation. Notice that the scanner has two arms, one mobile and the other used as a mechanical support, passing through the $x y$ plane.

with $*$ the complex conjugation, $k$ the propagation constant and $\hat{k}$ the direction of propagation of each plane wave composing the wavefront. The integration over $\hat{k}$ is limited to the directions covered by the auxiliary surfaces, in this case only the $2 \pi$ steradian of the upper half space, i.e., the region spanned by the field scanner described in Sec. IV.

\section{EXPERIMENTAL SETUP}

Experimental tests were based on the setup shown in Fig. 3. It consisted of a reverberation chamber, of dimensions $6 \times$ $3.5 \times 2.5 \mathrm{~m}^{3}$, made of galvanised steel. A mechanical stirrer is present but not rotated during the tests, in order to have a static environment, which is a fundamental requirement in order to ensure the best performance in a complex environment [20]. A stirrer can still be of practical interest, as it effectively reduces degeneracies in the modal response of a medium, as due to symmetries.

The electric Green's function, underpinning the synthesis of the excitation signals discussed in Sec. II, is sampled thanks to a dielectric robot. The robot was designed in order to weakly perturb a propagation medium as it moves a probe over a hemispherical surface [21]. The radius of this surface is equal to $1.18 \mathrm{~m}$ and can be modified manually, by operating on the probe holder, in order to sample the electric Green's function over two concentric surfaces, as required by Sec. II.

The electric field tangential to the hemispherical surface was sampled with an electro-optical probe, manufactured by Enprobe (model EFS-105). These probes have a flat frequency response up to $3 \mathrm{GHz}$ and are sensitive to phase shifts, making them capable of capture information necessary for time-domain analysis. They are also linearly polarized, with a cross-polarization rejection higher than $40 \mathrm{~dB}$. The $\vartheta$ and $\varphi$ tangential scalar components of the electric field were sampled by repeating the scan after rotating the probe by 90 degrees.

Choosing a frequency range for these experiments is a critical decision. On the one hand, increasing frequency enables 


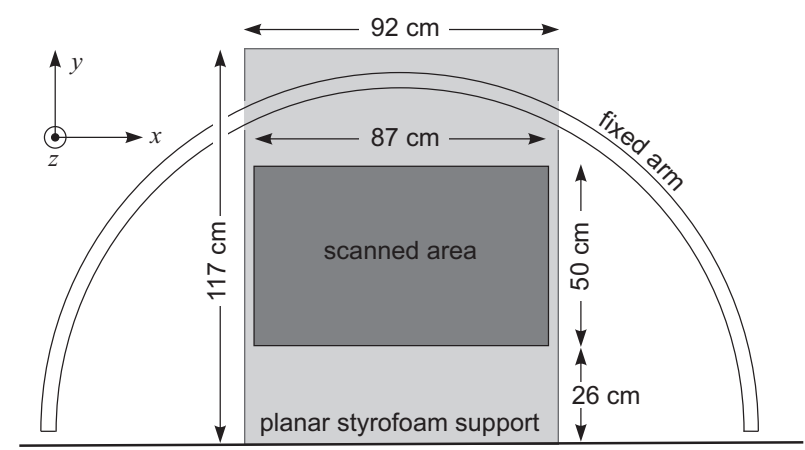

Fig. 4: Layout of the scanned planar area, as part of the $x y$ plane.

the synthesis of finer focal spots, which would be interesting to test as capable of higher spatial resolution; on the other hand, the robot would introduce stronger perturbations and thus risk degrading the coherence of the field [21]: it would then be harder to understand if errors were due to limitations in GTR or perturbations from the robot. Moreover, the frequencies should be chosen as to ensure that the chamber stays diffusive, i.e., avoiding extended frequency regions where no resonant mode is accessible. For the above chamber, a minimum frequency of about $500 \mathrm{MHz}$ can be considered as safe in this respect, as modal overlapping, partly enabled by moderate losses, ensures that enough degrees of freedom are available [22]. These considerations should be regarded as a conservative requirement, being based on the behaviour of a reverberation chamber excited by a single harmonic signal. As TR applications cover bandwidths that would span a large number of resonant modes of a chamber, standard requirements for modal overlapping can be relaxed. Losses also have an impact on the efficiency of TR and GTR, as they directly control the intensity of the generated wavefront for a given input peak power [10].

For our experiments we settled for the bandwidth $1.5 \div 2.5$ $\mathrm{GHz}$ as a good compromise between spatial resolution and robot perturbation. The two hemispherical surfaces were then set $3.5 \mathrm{~cm}$ apart, i.e., about a quarter of wavelength at $2 \mathrm{GHz}$; this choice ensures that for all the frequencies considered the sampled field distributions will be maximally uncorrelated, in order to avoid any redundancy [23]. The angular sampling was set to 2 degrees, corresponding to a spatial sampling of about 4 $\mathrm{cm}$, in order to have data close enough to ensure a meaningful spatial interpolation. Robot movements are fully automated and controlled through an optical-fiber interface linked to a personal computer.

A single excitation antenna was used, close to the stirrer, as visible in Fig. 3. The antenna was a conical monopole, about $30 \mathrm{~cm}$ about the metallic floor, set obliquely. In fact, because of the diffusive nature of waves propagating in the chamber, the features of the antenna have no importance on the final results, though different excitation signals would then be needed (see Sec. II); the orientation chosen was in fact motivated by the need to avoid any symmetry in the setup, which could reduce the number of degrees of freedom on the field.
Transfer functions between the antenna and the field probe were measured by means of a vector network analyzer (Rohde \& Schwarz ZVB8). Frequency sampling needs to be chosen carefully, in order to avoid time aliasing, due to the relatively long relaxation time of the chamber, around $1 \mu \mathrm{s} ; 5000$ frequency samples were acquired, with a step of $200 \mathrm{kHz}$, thus a maximum time window of $5 \mu \mathrm{s}$.

The data thus collected were used for the synthesis of the excitation signals, using (2). Equally important are the data needed in order to image the field distributions generated by these excitation signals. In order to avoid scanning a surface for each excitation signal used, as it would be necessary for a time-domain excitation, we rather used a two-step approach, requiring a post-processing phase. The idea is again to sample the electric Green's function but this time over the surface over which the wavefront generated with GTR is to be imaged. These samples are therefore no longer used for synthesis, but rather for checking the accuracy of theoretical predictions. Knowing these samples, later referred to as $\Pi(\boldsymbol{r}, \omega)$, the field distributions can be computed as explained in Sec. V.

To this effect, a styrofoam block was introduced, oriented as to have one of its surfaces tangent to the $x y$ plane, hereafter referred to as the planar monitor. It was used for holding the electro-optical probe over a Cartesian grid graved over its surface; the sampling distance was set to $3.1 \mathrm{~cm}$. The region shown in Fig. 4 was manually scanned, measuring the two tangential polarizations at each point. The normal component was not measured, since it was already proven that timereversed wavefronts excited in a reverberation chamber present the same accuracy in the three field components, independently of the source used [24].

\section{IMAGING WAVEFRONTS}

Given the transfer functions $\boldsymbol{\Pi}(\boldsymbol{r}, \omega)$ between the excitation antenna and a position $r$ over the planar monitor, the timedomain field generated by an excitation signal $x(t)$ with a Fourier spectrum $X(\omega)$ can be retrieved by computing

$$
\boldsymbol{E}(\boldsymbol{r}, t)=\frac{1}{2 \pi} \int \mathrm{d} \omega \boldsymbol{\Pi}(\boldsymbol{r}, \omega) X(\omega) \mathrm{e}^{\mathrm{j} \omega t} .
$$

The analysis of the accuracy of the wavefronts generated by GTR is based on sources with $\psi_{s}=40$ degrees (see Sec. III); Sec. VI-C also considers the case $\psi_{s}=10$ degrees. The angular distributions of these two wavefronts, i.e, the modulus of their PWS, are shown in Fig. 5.

Apart from Sec. VI-C, all the results involve wavefronts evolving in time as Gaussian pulses with $B_{e}=500 \mathrm{MHz}$, in order to ensure a high contrast between the coherent wavefronts and the background diffuse contribution. The rationale is to create the conditions for judging unambiguously if the wavefronts generated with GTR do indeed evolve as in freespace conditions. The effects of reducing the bandwidth and/or the focusing power are discussed in Sec. VI-C, where they are shown to be two facets of the same problem.

In this section we first consider the generation of a Gaussian wavefront propagating along the direction $\vartheta=\varphi=90$ degrees, towards the floor. Its polarization is set to be tangent 

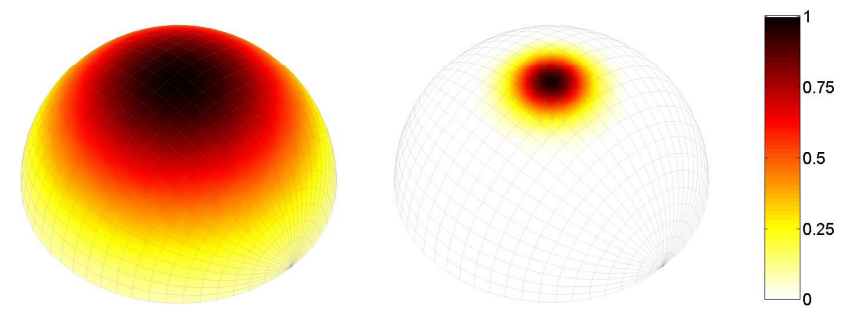

Fig. 5: Angular distribution of the Gaussian beams used throughout the experimental validation: (a) $\psi_{s}=40$ degrees, (b) $\psi_{s}=10$ degrees.
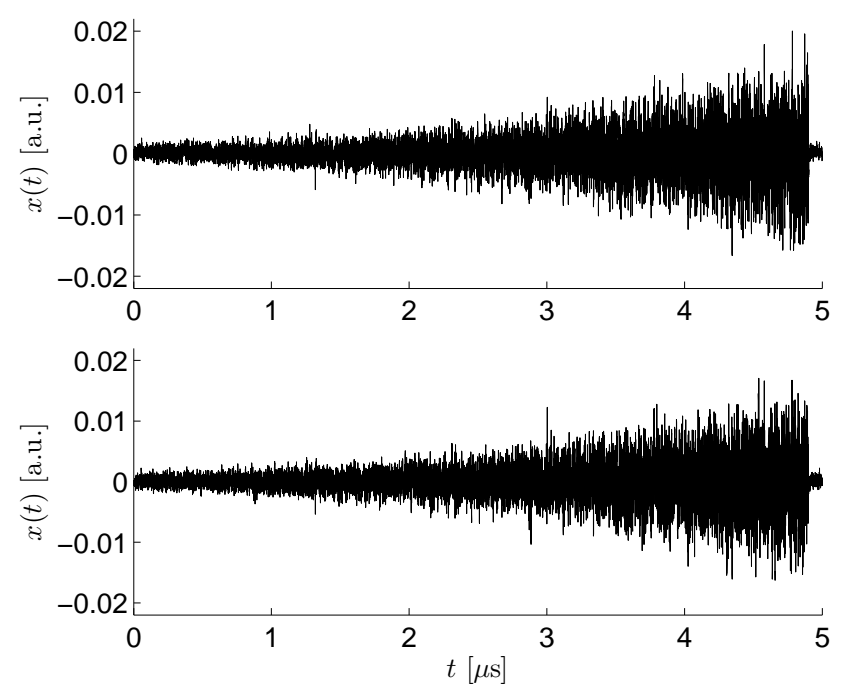

Fig. 6: An example of excitation signals obtained with GTR, for the wavefronts shown in Fig. 11, for a normal incidence along the $y$ axis (top figure) and an oblique one, tilted by 40 degrees along the $x y$ plane (bottom figure).

to the planar monitor, i.e., to the plane $x y$, or a $\hat{\varphi}$-polarized wave. The focus is set in the center of the imaged region.

Combining the data collected with the setup described in Sec. IV together with the theoretical wavefront distribution at the auxiliary surfaces $\Sigma_{i}$ as in (2), we obtained the excitation signals expected to reproduce the wavefront within the chamber. Two examples of such signals are presented in Fig. 6 , encoding the information needed for generating focusing wavefronts along two directions of incidence. They share the same envelope, as this is directly controlled by the relaxation response of the medium.

Rather than synthesizing these signals in time domain and injecting them into the chamber through the antenna, we proceeded as explained at the beginning of this section. The field distribution over the planar monitor was then computed at a few time instants, in order to observe the focusing wavefront as it approaches the focal region.

Figs. 7 and 8 present the evolution of the $E_{x}$ component of the field, respectively theoretically and experimentally. The results closely agree both in the time and space evolution.
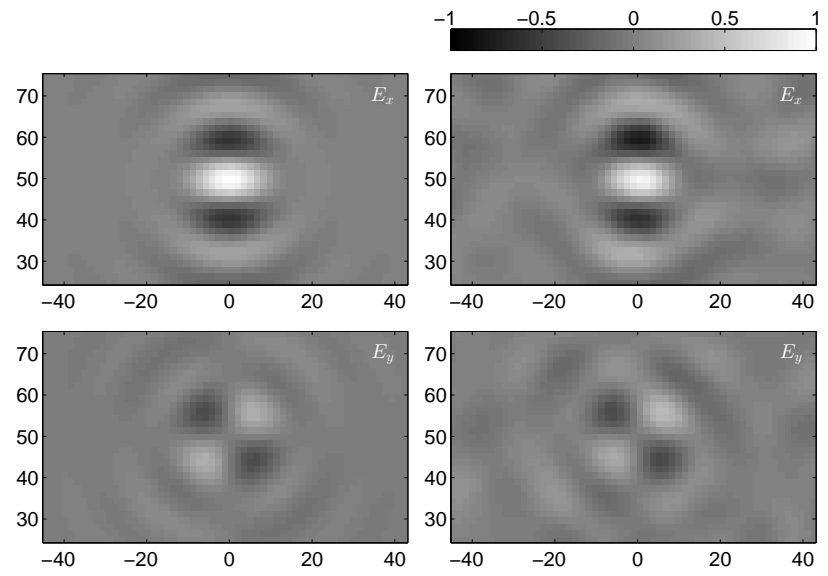

Fig. 9: Distributions of the horizontal $\left(E_{x}\right)$ and vertical $\left(E_{y}\right)$ field components expected (left column) and measured (right column) at $t=0$. Linear correlation between the theoretical and experimental results is $97 \%$ for $E_{x}$ and $94 \%$ for $E_{y}$. Results normalized to the peak of the norm of the respective electric fields.

The existence and intensity of the background fluctuations can be appreciated in Fig. 8 for $t=-5 \mathrm{~ns}$, when the wavefront is expected to be negligible. The field distribution then resembles closely a speckle distribution, with cells about half a wavelength wide.

The coherent evolution of the generated wave is further confirmed in Fig. 8 at $t=3$ ns, where the distribution matches that of a wavefront reflected by the floor and moving back upwards. The divergence and delay are in good accordance with the total distance travelled from the focus.

Hereafter only results for $t=0$ will be discussed. The focal instant can be regarded as the most important one, as it shows the wavefront that would impinge onto an system if it was present in the chamber in the first place, also during the sampling of Green's functions. It would be impossible to image the impinging field distribution in this last case, since the scattered field would overlap with it; tests carried out in the conditions described in this paper therefore provide precious information for the definition of applications that could benefit from GTR performance.

Fig. 9 shows the two measured field components for $t=0$, compared to those expected for the convergent version of the Gaussian wavefront. Data match very closely, providing an explanation for our showing only the horizontal component $E_{x}$ of the electric field, which is also the dominant one.

\section{WAVEFRONT FIDELITY}

Having verified the accurate reproduction of the convergent wavefront, it is fundamental to assess whether GTR can generate it with the same accuracy even when its focus and directions of arrival are changed. The theoretical derivations in [9] concluded that the homogeneous characteristics of diffusive media should ensure a comparable reproduction for any set of parameters, at least on average. 

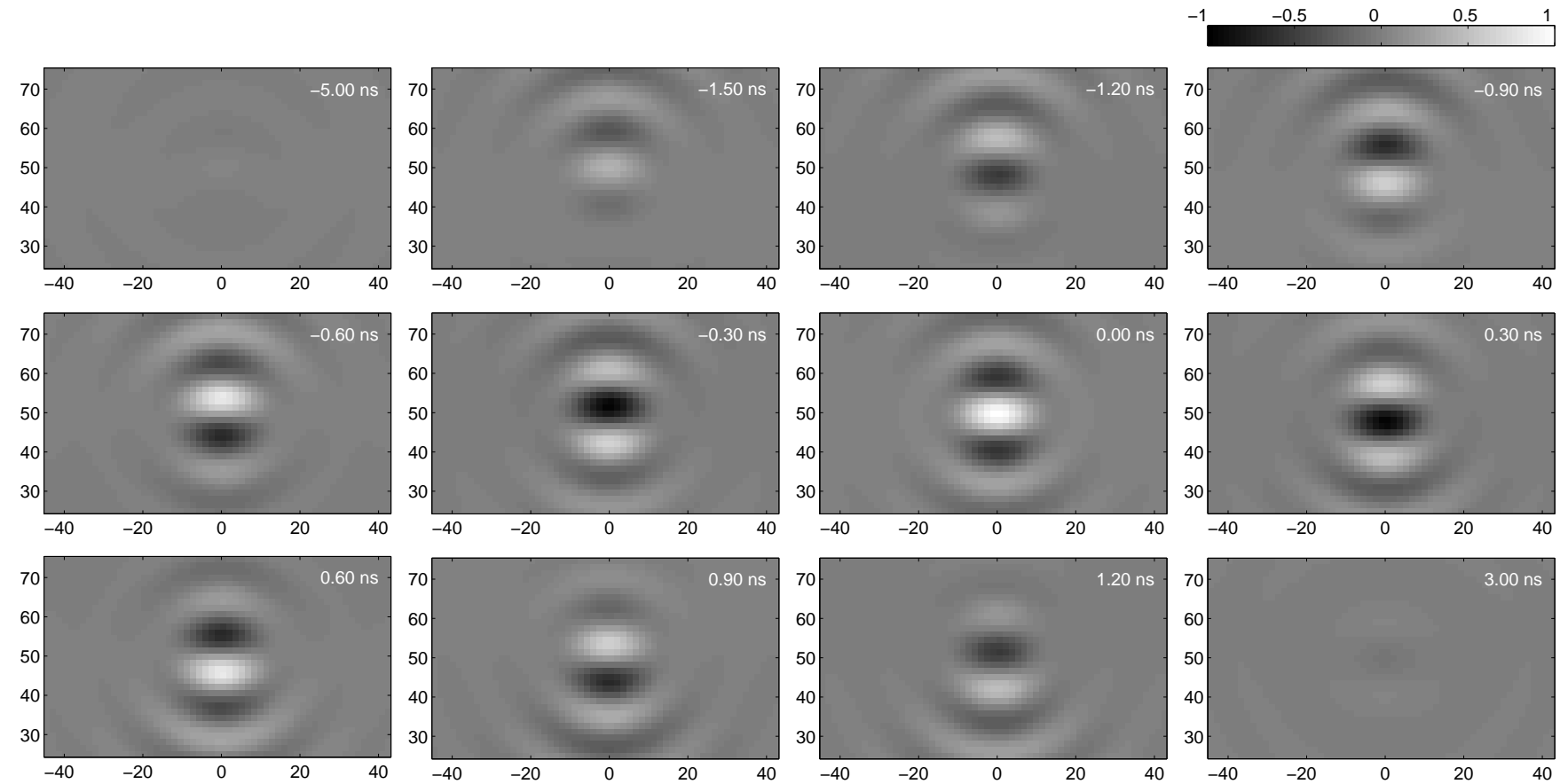

Fig. 7: Theoretical distributions of the horizontal field component $E_{x}$ expected over the region shown in Fig. 4, for $\psi_{s}=40$ degrees, $B_{e}=500 \mathrm{MHz}$. Results normalized to the peak of the norm of the electric field at $t=0$.
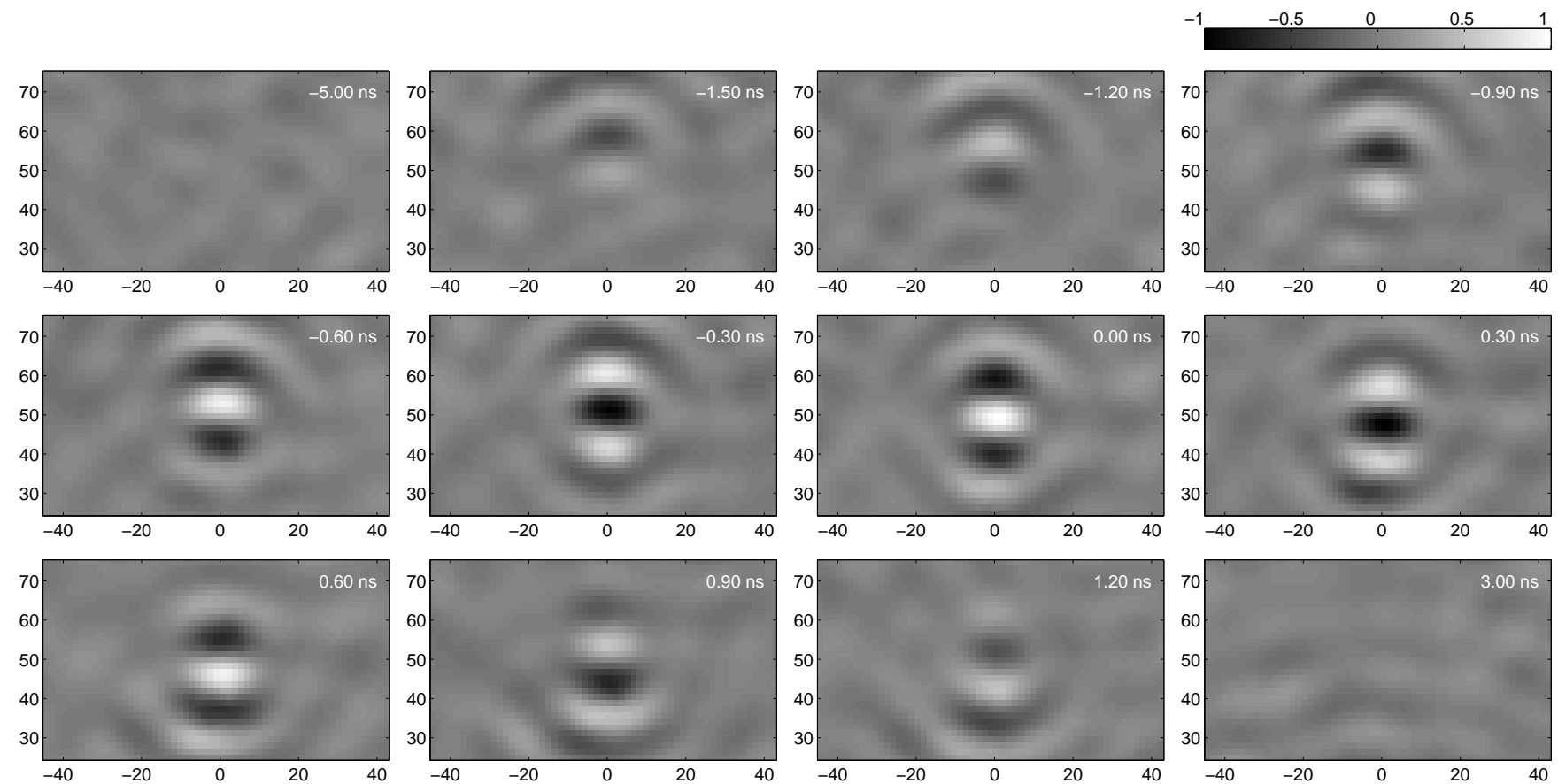

Fig. 8: Field distributions of $E_{x}$ measured over the region shown in Fig. 4 , for $\psi_{s}=40$ degrees, $B_{s}=500 \mathrm{MHz}$,. At $t=-5$ $\mathrm{ns}$, only background fluctuations are visible; at $t=3 \mathrm{~ns}$ the wavefront propagates back after reflecting on the metallic floor. Results normalized to the peak of the norm of the electric field at $t=0$. 

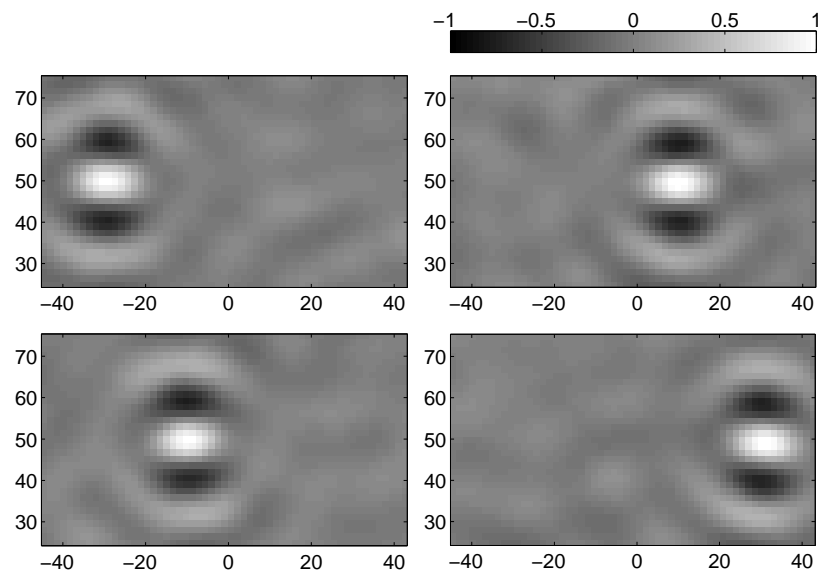

Fig. 10: Measured field distributions for $E_{x}$, for $\psi_{s}=40$ degrees, $B_{e}=500 \mathrm{MHz}$ and $t=0$, as the position of their focus is displaced horizontally, by $20 \mathrm{~cm}$ steps. Linear correlation between the four field distributions is above $95 \%$. Results normalized to the peak of the norm of the electric field at $t=0$ for the original central position shown in Fig. 9.

\section{A. Moving the focal spot}

The field distribution observed at $t=0$ was studied as the position of the focal spot changes. Four positions were considered, following a sequence of $20 \mathrm{~cm}$ horizontal displacements. The results are shown in Fig. 10 for the dominant field component, $E_{x}$. An excellent fidelity was found, with both the field structure and its amplitude practically unchanged.

\section{B. Changing direction of arrival}

The next test was to verify the wavefront fidelity when the direction of arrival changes. Since rotations would also affect the orientation of the main polarization, it is now necessary to study the polarization vector of the field. Five directions were considered, for $\varphi$ changing from -40 to 40 degrees around the initial direction, in steps of 20 degrees.

The results are shown in Fig. 11, where theoretical field distributions are compared with those found in practice. The agreement is again satisfying, with the focal regions practically identical to theoretical predictions. As discussed in Sec. VI-C, the regions outside the focal region are more strongly affected by random fluctuations inherent in the use of a diffusive medium; as a result, the agreement is not perfect outside the focal region.

\section{Focusing and contrast}

Previous results were obtained in optimal conditions, choosing wavefronts both confined in time (wide bandwidth) and in space (high values of $\psi_{s}$ ). The reason for the optimal performance for this case can be understood by recalling that wavefront-related quantities (e.g., received signals) present a mixture of coherent components, i.e., the wavefront meant to be generated, and incoherent ones, i.e., the random background fluctuations observed in the field distributions. It was shown
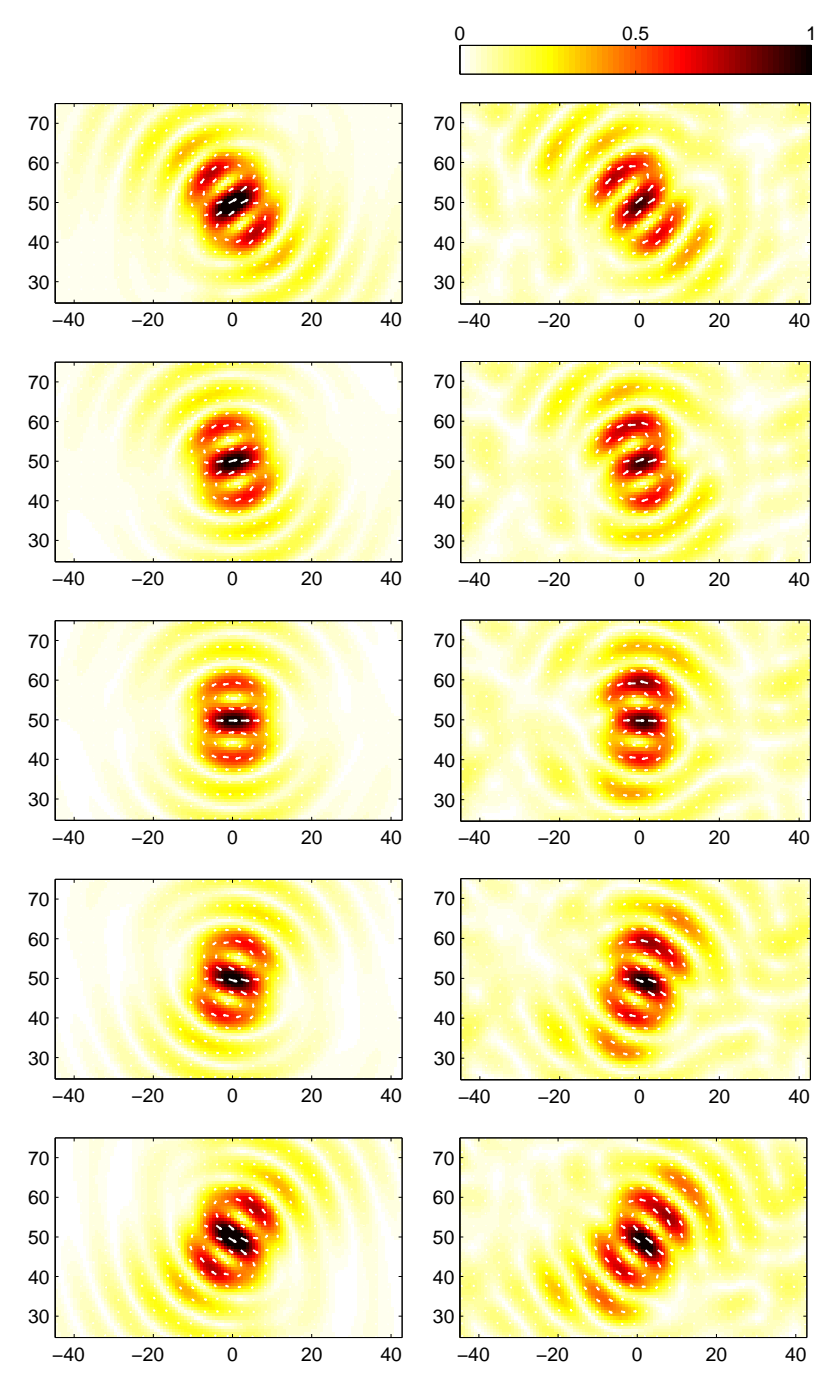

Fig. 11: Vector field distributions (theoretical on the left and measured on the right), for $\psi_{s}=40$ degrees, $B_{e}=500$ $\mathrm{MHz}$ and $t=0$, for wavefronts impinging along a direction of arrival $\hat{\boldsymbol{r}}_{\text {inc }} \in x y$, rotated by 20 degree steps, from -40 degrees to 40 degrees with respect to the $\hat{\boldsymbol{y}}$ axis. Results are normalized to the peak value of each field distribution. Linear correlation coefficients between experimental and theoretical spatial distributions of the field norm are 95\%, 94\%, 93\%, $93 \%$ and $92 \%$, from top to bottom.

in [25] that the ratio between the average energy densities conveyed by these two components is fixed by the statistical properties of the medium itself; e.g., for a diffusive medium it is equal to one.

This result has a very practical implication: if the coherent energy is fixed, then depending on how it is shaped, the instantaneous power can take very different values. For instance, if energy is shaped into a short pulse, the peak to energy ratio will be maximized, leading to a peak in the coherent component, making it stay well above the background fluctuations. On the contrary, if a narrower bandwidth is chosen, the energy will be spread over a larger time interval, 

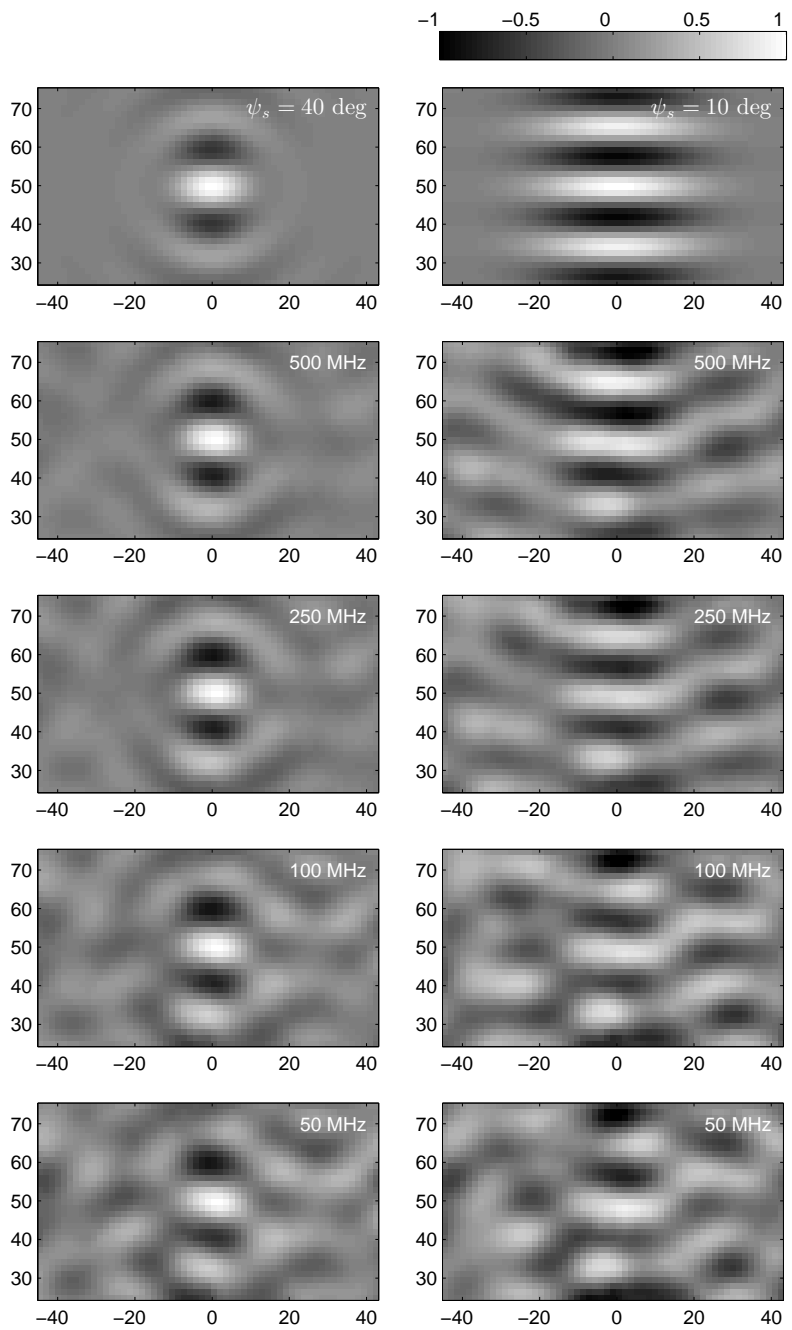

Fig. 12: Comparison between $E_{x}$ field distributions obtain for two Gaussian beams, with $\psi_{s}=40$ degrees (left column) and $\psi_{s}=10$ degrees (right column), as the equivalent bandwidth $B_{e}$ changes. Notice how the focal region is hardly affected for $\psi_{s}=40$ degrees, even for a relatively narrow bandwidth, as opposed to the case for $\psi_{s}=10$ degrees. Results are normalized to the peak value of each field distribution. Linear correlation coefficients between experimental and theoretical spatial distributions are, from top to bottom, 97\%, 95\%, 94\% and $93 \%$, for $\psi_{s}=40$ degrees and $94 \%, 93 \%, 87 \%$ and $73 \%$, for $\psi_{s}=10$ degrees.

hence a lower peak instantaneous power, with a subsequent risk of having it drowned into the background fluctuations.

This reasoning leads to conclude that both time and spatial focusing play the same role: weakly converging wavefronts, somewhat closer to plane waves over regions spanning several wavelengths, also spread energy over space. Examples going in this sense are shown in Fig. 12, where wavefronts with different combinations of bandwidth and angular convergence are considered. For the same bandwidth, the focal region can be shown to be about 6 times larger when $\psi_{s}=10$ degrees than for $\psi_{s}=40$ degrees. As a result, in order to maintain a similar contrast between the coherent wavefront and the background in the two cases, a bandwidth about 6 times larger is required for $\psi_{s}=10$ degrees. This conclusion is consistent with the observation of similar background fluctuations in the results for $B_{e}=250 \mathrm{MHz}$ (for $\psi_{s}=10$ degrees) and $B_{e}=50$ $\mathrm{MHz}$ (for $\psi_{s}=40$ degrees).

In short, bandwidth and angular divergence do not put into question the ability of GTR to generate accurate wavefronts, but rather need to be regarded as design parameters that impact the margin between the wavefront and the background fluctuations.

\section{CONCLUSIONS}

GTR was applied to a reverberation chamber, as a way of generating coherent focusing wavefronts. These were proven to create focal regions very close to those theoretically computed. Moreover, their features were practically independent of their direction of arrival or position.

The results shown make clear that the appearance of the wavefronts is not due to a local compensation of the medium response, but corresponds to an actual wavefront interacting with the medium boundaries, as proven by the reflections shown in Fig. 8.

GTR is therefore an effective way of generating arbitrary focusing wavefronts with a single antenna. Its main limitations are the need for a low-perturbation field scanner and sufficiently high space-time focusing, which make it better suited to the generation of pulsed wavefronts.

\section{REFERENCES}

[1] D. Cassereau and M. Fink, "Time-reversal of ultrasonic fields. III Theory of the closed time-reversal cavity," IEEE Transactions on Ultrasonics, Ferroelectrics and Frequency Control, vol. 39, no. 5, pp. 579592, 2002.

[2] P. Roux, B. Roman, and M. Fink, "Time-reversal in an ultrasonic waveguide," Applied Physics Letters, vol. 70, p. 1811, 1997.

[3] W. Kuperman, W. Hodgkiss, H. Song, T. Akal, C. Ferla, and D. Jackson, "Phase conjugation in the ocean: Experimental demonstration of an acoustic time-reversal mirror," The Journal of the Acoustical Society of America, vol. 103, p. 25, 1998.

[4] M. Fink and C. Prada, "Acoustic time-reversal mirrors," Inverse Problems, vol. 17, p. R1, 2001.

[5] P. A. Naylor and N. D. Gaubitch, Speech dereverberation. Springer Science \& Business Media, 2010.

[6] E. W. Start, Direct sound enhancement by wave field synthesis. TU Delft, Delft University of Technology, 1997.

[7] H. Moussa, A. Cozza, and M. Cauterman, "Directive wavefronts inside a time reversal electromagnetic chamber," in Electromagnetic Compatibility, 2009. EMC 2009. IEEE International Symposium on, Aug 2009, pp. $159-164$.

[8] - "Experimental demonstration of directive pulsed wavefront generation in reverberation chambers," Electronics Letters, vol. 46, no. 9, pp. 623-624, 2010.

[9] A. Cozza, "Emulating an anechoic environment in a wave-diffusive medium through an extended time-reversal approach," Antennas and Propagation, IEEE Transactions on, vol. 60, no. 8, pp. 3838-3852, 2012.

[10] H. Vallon, A. Cozza, F. Monsef, and A. S. Chauchat, "Time-reversed excitation of reverberation chambers: Improving efficiency and reliability in the generation of radiated stress," IEEE Transactions on Electromagnetic Compatibility, vol. 58, no. 2, pp. 364-370, April 2016.

[11] D. Hill, "Plane wave integral representation for fields in reverberation chambers," IEEE Transactions on Electromagnetic Compatibility, vol. 40, no. 3, pp. 209 -217, aug 1998.

[12] H. Kuttruff, Room acoustics. Taylor \& Francis, 2000. 
[13] C. Holloway, D. Hill, J. Ladbury, P. Wilson, G. Koepke, and J. Coder, "On the use of reverberation chambers to simulate a Rician radio environment for the testing of wireless devices," IEEE Transactions on Antennas and Propagation, vol. 54, no. 11, pp. 3167 -3177, 2006.

[14] C. Draeger, J. Aime, and M. Fink, "One-channel time-reversal in chaotic cavities: Experimental results," The Journal of the Acoustical Society of America, vol. 105, p. 618, 1999.

[15] A. Derode, P. Roux, and M. Fink, "Robust acoustic time reversal with high-order multiple scattering," Physical Review Letters, vol. 75, no. 23, pp. 4206-4209, 1995.

[16] M. Born and E. Wolf, "Principles of opticspergamon press," New York, 1980.

[17] R. Collins and F. Zucker, Antenna Theory. McGraw-Hill, New York, 1969.

[18] T. Hansen and A. D. Yaghjian, Plane-wave theory of time-domain fields. IEEE Press, 1999

[19] A. Cozza and F. Monsef, "Layered electric-current approximations of cylindrical sources," Wave Motion, vol. 64, pp. 34-51, 2016.

[20] — "On the influence of medium statistics on the robustness of time-reversal transmissions," IEEE Antennas and Wireless Propagation Letters, vol. PP, no. 99, pp. 1-1, 2016.

[21] A. Cozza, F. Masciovecchio, C. Dorgan, M. Serhir, F. Monsef, and D. Lecointe, "A dielectric low-perturbation field scanner for sensitive environments," Antennas and Propagation, IEEE Transactions on, 2016, under review.

[22] A. Cozza, "The role of losses in the definition of the overmoded condition for reverberation chambers and their statistics," IEEE Transactions on Electromagnetic Compatibility, vol. 53, no. 2, pp. 296-307, May 2011.

[23] D. Hill and J. Ladbury, "Spatial-correlation functions of fields and energy density in a reverberation chamber," IEEE Transactions on Electromagnetic Compatibility, vol. 44, no. 1, pp. 95-101, 2002.

[24] A. Cozza and H. Moussa, "Enforcing deterministic polarisation in a reverberating environment," Electronics Letters, vol. 45, no. 25, pp. 1299-1301, 2009.

[25] A. Cozza and F. Monsef, "Multiple-source time-reversal transmissions in random media," Antennas and Propagation, IEEE Transactions on, vol. 62, no. 8, pp. 4269-4281, Aug 2014. 